\title{
Alternative approaches to generalized sidelobe canceler
}

This paper was downloaded from TechRxiv (https://www.techrxiv.org).

\section{LICENSE}

CC BY 4.0

SUBMISSION DATE / POSTED DATE

04-10-2021 / 13-10-2021

CITATION

Phan, Son (2021): Alternative approaches to generalized sidelobe canceler. TechRxiv. Preprint. https://doi.org/10.36227/techrxiv.16732288.v1

$\mathrm{DOI}$

10.36227/techrxiv.16732288.v1 


\title{
Alternative approaches to generalized sidelobe canceler
}

\author{
Phan Le Son
}

\begin{abstract}
The generalized sidelobe canceler (GSC) decomposes the beamforming into two paths: The upper path is to preserve the desired signal, the lower path is to suppress the desired signal. The GSC design was proposed in the time-domain, then it is easily applied for the broadband beamforming. From beampattern viewpoint, we propose alternative approaches to the GSC, the new algorithms mainly modify in the lower path, that is, instead of using the blocking matrix to suppress the desired signal, we design a beamformer that contains the nulls at the look direction and some other directions. We claim that the beamforming with a null at look direction performs a similar function to the blocking matrix. Moreover, the GSC subtracts two beampatterns to suppress the side-lobes, this amplifies noise when the sidelobes of the upper path beampattern are not identical to the lower path beampattern, especially at the null positions of the upper path beampattern. To tackle this issue, we insert those nulls to the lower path beampattern to increase the similarity of the beampatterns at two paths. By doing so, we propose two variants of the GSC, one is the fixed beamforming where the adaptive filter in the lower path of the GSC is removed and the other is the adaptive beamforming where only one adaptive filter is used.
\end{abstract}

Index Terms-Fixed beamforming, adaptive beamforming, superdirective beamforming, differential microphone array.

\section{INTRODUCTION}

Array signal processing (ASP) [1], [2] has been widely employed in diverse areas such as acoustics [3], [4], radiointerferometry [5], [6], radar and sonar systems [2], [7], wireless networks [8], [9], [10] and medical imagery [11]. Beamforming is an important topic in ASP [1], that is the process of performing spatial filters to preserve the signal from directions of interest while suppressing interfering signals and noise arriving from other directions. A fixed beamformer is a beamformer whose coefficients are independent on the measurement signals, it normally uses to suppress stationary noise. On the other hand, an adaptive beamformer is the beamformer whose coefficients depend on or adapt to measurement signals, it is capable of suppressing interferences but it suffers from computationally intensive and signal distortions. In many applications, the bandwidth of signals of interest spreads over several octaves, therefore the characteristics of the beamformer should be invariant over frequency of interest, which is achieved via the so-called broadband beamforming.

For instance, if noise fields are stationary and well-defined, then using fixed beamforming is a reasonable choice. Most fixed beamformer have a closed-form expression for beamformer coefficients. For example, the delay and sum beamforming (DSB) is the simplest beamforming technique where

The author is with the group of Digitale Signalverarbeitung, Elektrotechnik und Informationstechnik, Technische Universität Kaiserslautern, Germany. Email:phan@eit.uni-kl.de the signal of sensors are delayed to align in phase and then be summed [12]. Actually, beamformer coefficents of DSB are the optimal solution for the noise suppression when only white noise is immersed in the signal. Later, superdirective beamforming (SD) [13] was proposed to consider the presence of diffuse noise. However, at low frequencies, the SD beamforming amplifies white noise. Therefore, the regularization of $\mathrm{SD}$, considering white noise, is commonly used in practical applications. For acoustic signals, differential microphone arrays (DMAs) are used in the variety of applications. Conventional DMA is based on the spatial derivatives of the acoustic pressure field [14], [15], [16]. Since the sensor spacing of the DMA is much smaller than the acoustic wavelength, the DMA is small in size and can be easily mounted into other devices. On the other hand, based on the short-time Fourier transform (STFT), spatial filtering is applied to form a differential beamformer in each subband [17], [18], [19], [20]. The order of the differential beamformer could be designed by selecting the number of null-constraints and the type of differential beamformer could also be obtained by assigning the null positions and/or changing the optimization objective function such as maximum front-to-back ratio for a supercardioid microphone, maximum directivity index for a hypercardioid, etc.

The early adaptive beamforming technique is the linearly constrained beamforming [21], which minimizes the energy of array output with subject to distortion-less at look direction. Over the years, the general approach for this class of beamformer has been studied as the so-called linearly constrained minimize variance (LCMV) [12], [22], [23] where the objective is to minimize the variance of the array output, that enables to solve the problem from statistical viewpoint. In LCMV, the input signals are modeled to the covariance matrix computed via multiple frames observation, then the closedform expression for the solution is derived for some simple cases. An alternative approach to linearly constrained adaptive beamforming was proposed by Griffiths and Jim in [24], that is the general sidelobe canceler (GSC) beamformer. In GSC, they modified the constrained problem in [21] to unconstrained problem by introducing a fixed beamforming at the upper path and a blocking matrix at the lower path. The lower path signals are filtered by the adaptive FIR filters before be subtracted from the upper path signal. This scheme could preserve the signal of interest (SOI) in the upper path while removing the noise in the lower path. This simple approach could be easy to deploy in the real-time implementation. Besides, the GSC can be designed and processed in the time-domain, it is well-suited to broadband signal processing [25]. However, in practical applications, steering vector errors are inevitable, 
then the conventional blocking matrix could not block the target signal effectively, that degrades the performance of the GSC. To address those problems, robust adaptive beaformers based on GSC structure were proposed. In [26], [27], [28], they regard the blocking matrix as a set of filters where spatial and temporal frequency are merged to a single variable of the filter, then suppressing signals from a set of look direction at temporal frequency band is achieved by high-pass filter design. It is therefore flexible to expand the region of look direction to tolerate mismatches. However, these approaches reduce the degrees of freedom for interference cancellation. Later, Hoshuyama at al. in [29] added adapter filters inside the blocking matrix to minimize the leakage of target signal in the lower path. They restrict filter coefficients of the blocking matrix within a defined region associated with the tolerant region of look direction. This method increases the robust of the beamformer against steering vector errors without loss the degrees of freedom for interference reduction. On the other hand, instead of using the steering vector model, Gannot at al. in [30] considered acoustic transfer function ratios as the input of GSC and proposed a GSC solution which can be applied in a reverberating room. The transfer function ratios can be re-estimated if the acoustic environment has changed.

In general, most researches focus on blocking matrix design to remove the leakage effectively. However, subtracting structure in GSCs has another issue that has not addressed explicitly, e.g., noise/interference in the lower path and in the upper path are much different, then subtracting them does not suppress the noise/interference effectively (noise/interference in the upper path leaks to the final output). In this paper, we explain this issue from the beampattern perspective and propose a simple approach to overcome this problem. We regard the blocking matrix as the first order differential beamformings with a null at the look direction. Then, we analyze the difference between upper path's and lower path's beampatterns. In most of cases, the sidelobes of the upper path beampattern is much different with the lower path's beampattern and the difference is varying over frequency. Then, the subtraction can not remove the sidelobes effectively. Furthermore, the blocking matrix generates multiple almost identical beampatterns at the lower path, that is somewhat redundant.

To overcome those problems, we propose modified GSCs, that is, instead of using a blocking matrix in the lower path, we design a beamformer that has a null at look direction and some nulls at the nulls' position of the upper path beamforming. Some extra nulls at the lower path make the lower path beampattern is more similar to sidelobes of the upper path beampattern. Besides the shape of beampatterns, phases of beampatterns are also important that should be aware before subtracting the signals. For instance, the phase of the upper path and lower path beampattern are almost stable over the direction, we can use a fixed scale value in the lower path to correct the phase and amplitude of the subtracted signal. That is the case when the array layout is symmetric and the reference point is selected at the center of the array. As such, a fixed beamforming is proposed, referred to as a variant of the GSC. On the other hand, the phase difference is varying over the direction, we use an adaptive FIR filter at the lower path. Depending on the direction of interferences at an instance time, the FIR filter can scale the amplitude and adjust the phase of the lower path beampattern accordingly. Furthermore, to avoid target signal distortion at the final output, updating filter coefficients should be performed when the signal-tointerference ratio (SIR) is low [29], then we propose a simple method to change the step size of the adaptive FIR filter based on the energy of upper path and lower path signals. This adpative beamforming is also considered as another variant of the GSC. We briefly summarize the contributions of our work on beamforming design as follows:

1) We present some new insight into the GSC beamformer where we claim that the response of the blocking matrix is similar with that of first order Cardiod beampatterns with the null at look direction. From beampattern standpoint, we draw some disadvantages of the GSC.

2) We propose a fixed beamforming designed for a symmetric array layout. For this kind of array layout, we prove that the complex-valued beampattern of superdirective beamforming contains only the real part if we select the reference point at the center of the array. The beamforming designed by this approach has a frequencyinvariant mainlobe and the sidelobes are suppressed as much as possible.

3) For an arbitrary array layout, we propose an adaptive beamforming design. Its performance is not only superior to other methods, the computational complexity is also reduced. Moreover, this methodology gives the opportunity to combine multiple arrays to form a single expected beampattern.

4) We conduct the simulations and experiments to exterminate the proposed methods.

As the basis of our proposed approaches, we review the GSC beamforming in Section II. In Section III, we propose two alternative approaches to the GSC: A fixed beamforming with suppressed sidelobe (FBSS) and an adaptive beamforming with suppressed sidelobe (ABSS). The simulations and their results are provided in Section IV. The experiment is presented in Section V. Finally, the conclusion is drawn in Section VI.

\section{Generalized SIDELOBE CANCELER}

Consider an array with $M$ sensors, the GSC algorithm was proposed in the time-domain [24] where the signal of sensor $m$ at the discrete time index $n$ is defined by

$$
x_{m}(n)=s_{m}(n)+n_{m}(n), m=1, \ldots, M
$$

where $s_{m}(n)$ and $n_{m}(n)$ are the signal of interest (SOI) and noise, respectively. We can present the SOI at a sensor as the wave propagation from the source to the sensor

$$
s_{m}(n)=\mathbf{a}_{m} * \mathbf{s}_{0}
$$

where $\mathbf{a}_{m}$ is the transfer function of the wave propagation, $\mathbf{s}_{0}$ is the source signal and ' $*$ ' is the convolution operation. 
Let $\mathbf{x}_{A}(n)$ be the time-alignment towards the look direction of $\mathbf{x}(n)=\left[x_{1}(n), \ldots, x_{M}(n)\right]^{T}$, normally $\mathbf{x}_{A}(n)$ is implemented by fraction delays of the measurement signals. Fig. 1 plots the overview of GSC.

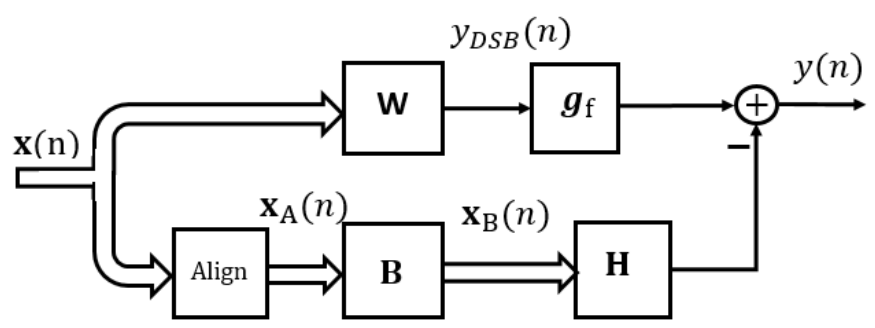

Fig. 1: Schematic description of the decomposition of the optimal weight vector into two orthogonal parts.

In the lower path, the SOI portion $s_{m}(n) m=1, \ldots, M$ is aligned and suppressed via a blocking matrix $\mathbf{B}$

$$
\mathbf{x}_{B}(n)=\mathbf{B} \mathbf{x}_{A}(n)
$$

where $\mathbf{x}_{B}(n)$ size of $M-1$ is the vector output of the blocking matrix, this signal vector should exclude the SOI. To do so, the blocking matrix $\mathbf{B}$ has to fulfill the following properties [24], [14]:

- The size of the matrix is $(M-1) \times M$

- The sum of all values in one row is zero

- The matrix has to be of rank $M-1$.

An example of blocking matrix for the case $M=4$ is

$$
\mathbf{B}=\left[\begin{array}{cccc}
1 & -1 & 0 & 0 \\
0 & 1 & -1 & 0 \\
0 & 0 & 1 & -1
\end{array}\right]
$$

The vector $\mathbf{x}_{B}$ is processed with adaptive FIR filters $\mathbf{H}=$ $\left[\mathbf{h}_{1}, \ldots, \mathbf{h}_{M-1}\right]^{T}$ and then be subtracted from the upper path's signal (e.g., the output of DSB) to get the noise-reduced signal, that is

$y(n)=\sum_{i=0}^{L-1} y_{D S B}(n-i) \mathbf{g}_{f}[i]-\sum_{i=1}^{M-1} \sum_{j=0}^{L-1}\left\{\mathbf{x}_{B}(n-j)\right\}[i] \mathbf{h}_{i}[j]$

where [.] indicates an element in a vector/matrix, $y_{D S B}($.$) is$ the output of the DSB, $g_{f}$ is a fixed FIR filter which ensures a specified gain and phase response for the output signal, $L$ is the length of FIR filters.

The early paper of GSC [24] used an iterative procedure to adaptive updating $\mathbf{h}_{i}, \forall i=1, \ldots, M-1$ in the least-meansquare sense

$$
\left\{\mathbf{h}_{i}[k]\right\}_{n+1}=\left\{\mathbf{h}_{i}[k]\right\}_{n}+\mu_{0} y(n) \mathbf{x}_{B}(n-k), k=1, \ldots, L
$$

where $\mu_{0}$ is the normalized step size computed from a small factor $\beta_{0}$ by

$$
\mu_{0}=\frac{\beta_{0}}{\sum_{i=1}^{M-1} \sum_{j=0}^{L-1}\left(\left\{\mathbf{x}_{B}(n-j)\right\}[i]\right)^{2}} .
$$

In summary, (2), (3) and (4) are the procedure of the GSC algorithm. The beamforming in the upper path and the blocking matrix are selected flexibility. However, how the selections affect the performance of GSC is not clearly explained.

From beampattern standpoint, we observe that one output of the blocking matrix $\mathbf{x}_{B}[m], m=0, \ldots, M-2$ is equivalent to the output of differential beamforming, that is delay and subtract signals. In case of a uniform linear array (ULA), its output contains $M-1$ almost identical first-order Cardioid beampatterns having null at look direction.

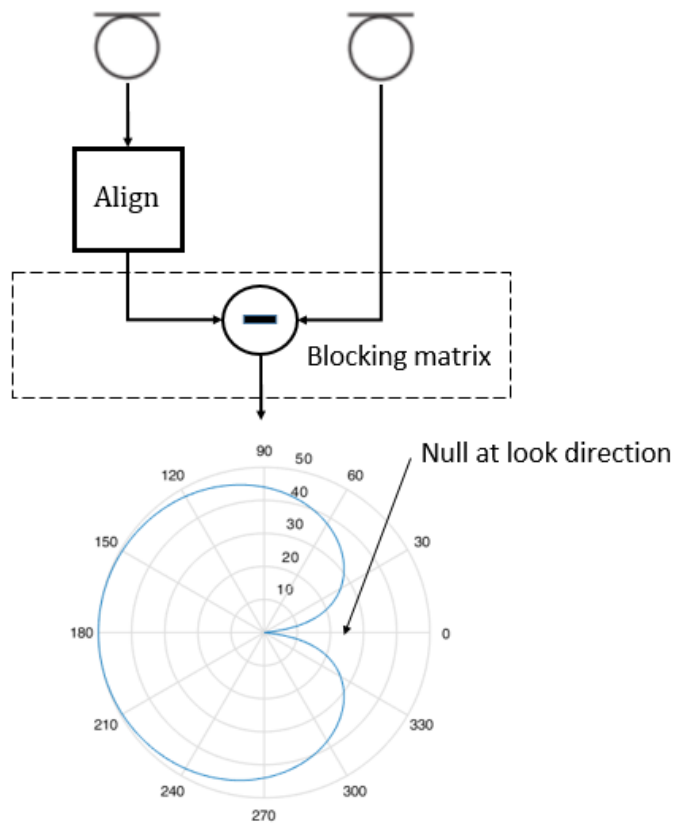

Fig. 2: An output of the blocking matrix.

As example for the ULA, at rotation frequency $\omega$, the $m t h$ output element of the blocking matrix in (1) to the signal at an incident direction $\theta$ is given by

$$
\begin{aligned}
& b_{m}(\omega, \theta)=e^{-j \omega \frac{m d_{H} \cos \theta}{c}} e^{-j T_{m}}-e^{-j \omega \frac{(m+1) d_{H} \cos \theta}{c}} e^{-j T_{m+1}} \\
& =e^{-j \omega \frac{m d_{H} \cos \theta}{c}} e^{j \omega \frac{m d_{H}}{c}}-e^{-j \omega \frac{(m+1) d_{H} \cos \theta}{c}} e^{j \omega \frac{(m+1) d_{H}}{c}} \\
& =e^{-j \omega m d_{H} \frac{\cos \theta-1}{c}}\left(1-e^{-j \omega d_{H} \frac{\cos \theta-1}{c}}\right)
\end{aligned}
$$

where $T_{m}$ and $T_{m+1}$ are the time delays at the sensor $m$ and $\mathrm{m}+1$, respectively, $d_{H}$ is the inter-distance of sensors, $c$ is the wave speed and $j$ is the imaginary unit. Then,

$$
\left|b_{m}(\omega, \theta)\right|=\left|1-e^{-j \omega d_{H} \frac{\cos \theta-1}{c}}\right|
$$

is the first order Cardioid beampattern with null at $\theta=0^{\circ}$ if $d_{H}<<\pi c / \omega$ [31], as illustrated in Fig. 2.

In the upper path, if a DSB is selected, then the gain of the beampattern obtains the maximum value at the look direction and less than that for other directions, e.g. Fig. 3 plots the DSB beampattern of ULA (endfire) with nine sensors (the inter-distance is $2.5 \mathrm{~cm}$ ) at different frequencies.

It is clearly seen that the sidelobes of the upper path's beampatterns are much different with the first-order Cardiod beampatterns in the lower path and the difference is varying 


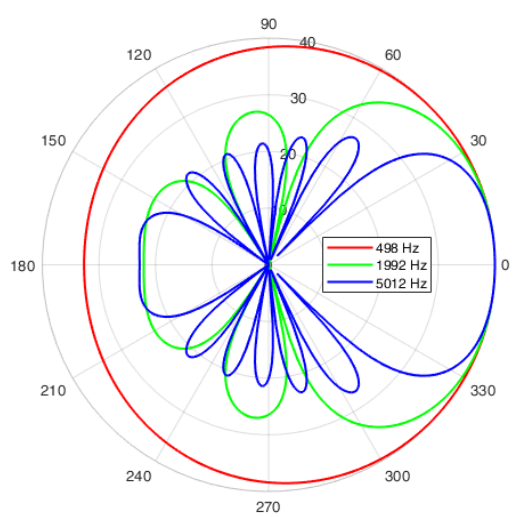

Fig. 3: Example for the DSB's beampatterns of the ULA at different frequencies.

over frequency. Therefore, the GSC needs to use adaptive filters that act as gain and phase controls for the first-order Cardiod beampatterns before subtracting them from upper path's beampattern. Also, the GSC uses $M-1$ almost identical beampatterns in the lower path, that is somewhat redundant, because scaling and sum many identical beampatterns are equivalent to scaling a single beampattern. That motivates us to modify the GSC in this study.

\section{Alternative APPROACHES to THE GENERALized SIDELOBE CANCELER}

The problem of subtracting different beampatterns in the GSC could be mitigated if we can design the lower path beamformer whose beampattern is almost identical to sidelobes of the upper path beampattern. That implementation is indeed feasible via the SD with multiple constraints where the nulls position can be inserted arbitrarily. To simplify our problem, in this paper, we restrict the spacing of sensors to be small compared to wavelengths of signal. That prevents the spatial aliasing issue which causes extra nulls in synthesized beampatterns.

At a narrow band in STFT, let $\mathbf{x}(\omega)=$ $\left[x_{1}(\omega), \ldots, x_{M}(\omega)\right]^{T}$ be a measurement vector of the array at the rotation frequency $\omega$, then the array response with beamforming is

$$
y(\omega)=\mathbf{w}(\omega)^{H} \mathbf{x}(\omega)
$$

where $\mathbf{w}(\omega)=\left[w_{1}(\omega), \ldots, w_{M}(\omega)\right]$ is the weight vector of the beamformer. The objective of SD with multiple constraints is to minimize the noise energy with subject to distortionless at the look direction and nulls at certain directions. Assume we want to design a beampattern having $N$ nulls at $\theta_{1}, \ldots, \theta_{N}$ and the distortionless at $\theta_{0}$, then the optimization problem is given as

$$
\begin{aligned}
& \underset{\mathbf{w}(\omega)}{\operatorname{minimize}} \mathbf{w}(\omega)^{H}(\boldsymbol{\Gamma}(\omega)+\mu \mathbf{I}) \mathbf{w}(\omega) \\
& \text { subject to } \\
& \quad \mathbf{w}(\omega)^{H} \mathbf{D}(\omega)=\mathbf{i}^{T}
\end{aligned}
$$

where $\mathbf{D}(\omega)=\left[\mathbf{d}\left(\omega, \theta_{0}\right), \mathbf{d}\left(\omega, \theta_{1}\right), \ldots, \mathbf{d}\left(\omega, \theta_{N}\right)\right]$ is the matrix size of $M \times(N+1)$ containing $N+1$ steering vectors, $\boldsymbol{\Gamma}(\omega), \mathbf{I}$ are the correlation matrix of diffuse noise and white noise, respectively, $\mu$ is a number deciding the noise model and $\mathbf{i}=[1,0, \ldots, 0]^{T}$ is a vector size of $N+1$.

Using the Lagrange multipliers method, the solution of SD with multiple constraints is given by [12]

$$
\mathbf{w}(\omega)=\frac{(\boldsymbol{\Gamma}(\omega)+\mu \mathbf{I})^{-1} \mathbf{D}(\omega)}{\mathbf{D}(\omega)^{H}(\boldsymbol{\Gamma}(\omega)+\mu \mathbf{I})^{-1} \mathbf{D}(\omega)} \mathbf{i} .
$$

For conciseness, we omit $\omega$ in the formulas in the remainder of this section whenever possible.

To manipulate the shape of beampattern, instead of using the DSB in the upper path, we also use a SD beamfomer in the upper path where the look direction is assured by the distortionless constraint, the sidelobe's shape and beamwidth are designed by nulls' position. For instance, the different phase response of the beampatterns at the lower and upper paths are almost equal over the spatial directions except the mainlobe region of upper path beampattern, we could apply a fixed filter to align the phase and scale the amplitudes before subtracting them. That enables us to use a fixed beamforming as an alternative approach to GSC. On the other hand, the different phase response of the beampatterns at the lower and upper paths is much varying over the spatial directions or we want to use more beamfomers at the lower path, we need to use an adaptive filter at the lower path, that is considered as a variant of GSC.

\section{A. Fixed beamforming with suppressed sidelobes}

At a narrow band in STFT, we design two beamformers, one is to construct the mainlobe with expected beamwidth (upper path beampattern) and the other is to replicate the sidelobes of the upper path beampattern (lower path beampattern). Then, we subtract the scale of lower path's signal from the upper path's signal to achieve a beampattern with suprressed sidelobes. Fig. 4 plots the block diagram of the fixed beamforming with suppressed sidelobes (FBSS).

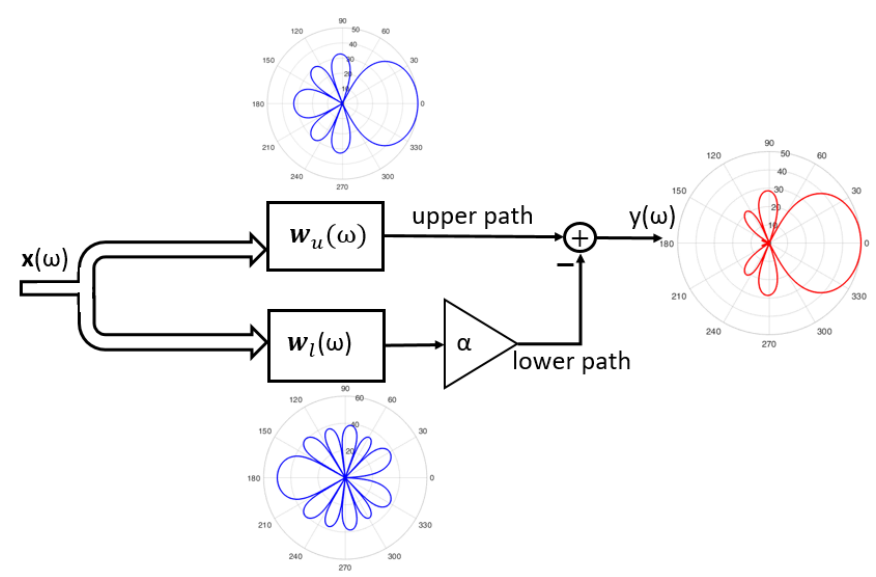

Fig. 4: Fixed beamforming with suppressed sidelobes. 
On the upper path, the beamformer is designed by

$$
\mathbf{w}_{u}=\frac{(\boldsymbol{\Gamma}+\mu \mathbf{I})^{-1} \mathbf{D}_{u}}{\mathbf{D}_{u}^{H}(\boldsymbol{\Gamma}+\mu \mathbf{I})^{-1} \mathbf{D}_{u}} \mathbf{i}_{u}
$$

where $\mathbf{D}_{u}=\left[\mathbf{d}\left(\theta_{0}\right), \mathbf{d}\left(\theta_{1}\right), \ldots, \mathbf{d}\left(\theta_{N}\right)\right]$ is the matrix size of $M \times(N+1)$ containing $N+1$ steering vectors, and $\mathbf{i}_{u}=$ $[1,0, \ldots, 0]^{T}$ is a vector size of $N+1$.

On the lower path, the beamformer is designed by

$$
\mathbf{w}_{l}=\frac{(\boldsymbol{\Gamma}+\mu \mathbf{I})^{-1} \mathbf{D}_{l}}{\mathbf{D}_{l}^{H}(\boldsymbol{\Gamma}+\mu \mathbf{I})^{-1} \mathbf{D}_{l}} \mathbf{i}_{l}
$$

where $\mathbf{D}_{l}=\left[\mathbf{d}\left(\theta_{m}\right), \mathbf{d}\left(\theta_{0}\right), \mathbf{d}\left(\theta_{1}\right), \ldots, \mathbf{d}\left(\theta_{N}\right), \ldots, \mathbf{d}\left(\theta_{N+K}\right)\right]$ is the matrix size of $M \times(N+K+2)$ containing $N+K+2$ steering vectors, $\theta_{m}$ is any direction that makes $\mathbf{w}_{l} \neq 0$ (e.g. $\theta_{m}$ is at the peak of sidelobes of upper path beampattern), $\theta_{N+1}, \ldots, \theta_{N+K}$ are the extra null directions in the upper path beampattern added by spatial aliasing effect when changing the frequency and $\mathbf{i}_{l}=[1,0,0, \ldots, 0]^{T}$ is a vector size of $N+K+2$.

The array response of the upper path beamforming at direction $\theta_{m}$ is

$$
\alpha=\mathbf{w}_{u}^{H} \mathbf{d}\left(\theta_{m}\right)=\mathbf{i}_{u}^{T} \frac{\mathbf{D}_{u}^{H}(\boldsymbol{\Gamma}+\mu \mathbf{I})^{-1} \mathbf{d}\left(\theta_{m}\right)}{\mathbf{D}_{u}^{H}(\boldsymbol{\Gamma}+\mu \mathbf{I})^{-1} \mathbf{D}_{u}} .
$$

Beamforming with suppressed sidelobes is designed by

$$
\mathbf{w}=\mathbf{w}_{u}-\alpha \mathbf{w}_{l} .
$$

Consider the class of arrays which are symmetric to the reference point, then the array response at the lower path and upper contain the real value only. For example, a ULA has a reference sensor at the center of array ( $M$ is an odd number), the steering vector is defined as

$\mathbf{d}(\theta)=\left[e^{\frac{j \omega(M-1) d_{H} \cos \theta}{c}}, \ldots, e^{\frac{-j \omega(M-1) d_{H} \cos \theta}{c}}\right]^{T}=\left[\mathbf{a}^{T}, 1, \mathbf{a}^{H}\right]^{T}$.

Similarly, we could present $\mathbf{D}_{u}$ as two conjunction parts

$$
\mathbf{D}_{u}=\left[\mathbf{A}_{u}^{T}, \mathbf{1}, \mathbf{A}_{u}^{H}\right]^{T}
$$

where 1 is the vector size of $N+1$ containing one for all element. The array response at the upper path,

$$
\mathcal{B}_{u}(\theta)=\mathbf{w}_{u}^{H} \mathbf{d}(\theta)=\mathbf{i}_{u}^{T} \frac{\mathbf{D}_{u}^{H}(\boldsymbol{\Gamma}+\mu \mathbf{I})^{-1} \mathbf{d}(\theta)}{\mathbf{D}_{u}^{H}(\boldsymbol{\Gamma}+\mu \mathbf{I})^{-1} \mathbf{D}_{u}}
$$

or

$$
\mathcal{B}_{u}(\theta)=\mathbf{i}_{u}^{T} \frac{\left[\mathbf{A}_{u}^{H}, \mathbf{1}, \mathbf{A}_{u}^{T}\right](\mathbf{\Gamma}+\mu \mathbf{I})^{-1}\left[\mathbf{a}^{T}, 1, \mathbf{a}^{H}\right]^{T}}{\left[\mathbf{A}_{u}^{H}, \mathbf{1}, \mathbf{A}_{u}^{T}\right](\mathbf{\Gamma}+\mu \mathbf{I})^{-1}\left[\mathbf{A}_{u}^{T}, \mathbf{1}, \mathbf{A}_{u}^{H}\right]^{T}}
$$

is a real number (see Appendix A).

We can obtain the similar result for the lower path beamforming. Then, this kind of array could be applied with the FBSS. From (11), it is clear that the FBSS is a beamforming that can be used to design a broadband beamforming where the mainlobe is maintained over frequency, while the sibelobes are suppressed as much as possible but may vary over frequency.

In a nutshell, we use (8), (9), (10) and (11) to design fixed beamforming for a symmetric array layout. The numerical simulation section will present some examples of this approach.

\section{B. Adaptive beamforming with suppresed sidelobes}

Analogous to the GSC, in this section, the proposed method is presented in the time-domain.

In case of spatial phase response of beamforming is complicated (vary over direction), we need a robust approach that can be adaptively updated the phase and gain of lower path beamforming before subtracting it from the upper path beamforming, which is called an adaptive beamforming with suppressed sidelobes (ABSS). Depending on the interference directions, at a narrow band, the appropriate gain and phase shift for the lower path beamforming is updated via an adaptive FIR filter.

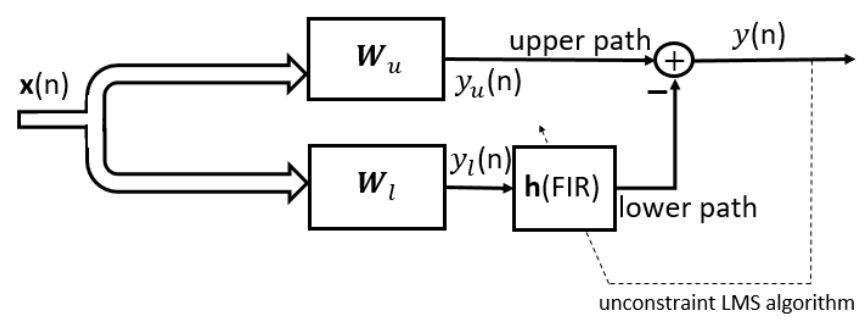

Fig. 5: Adaptive beamforming with suppressed sidelobes.

In Fig. 5, beamforming of the lower path and upper path are designed similarly to the FBSS, except that the coefficients are transformed to the time-domain as a set of fixed spatial filters, i.e., $\mathbf{W}_{u}, \mathbf{W}_{l}$ are the spatial filters' coefficients in the time-domain for the lower path and upper path, respectively.

Beamforming at the lower path is performed in the timedomain by spatial filtering

$$
y_{l}(n)=\sum_{i=0}^{M-1} \sum_{j=0}^{L_{1}-1}\{\mathbf{x}(n-j)\}[i] \mathbf{W}_{l}[i, j]
$$

where $L_{1}$ is the length of the spatial fitter of the lower path, $\mathbf{W}_{l}$ has dimension of $M \times L_{1}$, computed from (9) for the frequency of interest.

Beamforming at the upper path is performed in the timedomain by spatial filtering

$$
y_{u}(n)=\sum_{i=0}^{M-1} \sum_{j=0}^{L_{2}-1}\{\mathbf{x}(n-j)\}[i] \mathbf{W}_{u}[i, j]
$$

where $L_{2}$ is the length of the spatial fitter of the lower path (normally we set $L_{1}=L_{2}$ ), $\mathbf{W}_{u}$ has dimension of $M \times L_{2}$, computed from (8) for the frequency of interest.

The final output of ABSS is given as

$$
y(n)=y_{u}(n)-\sum_{i=0}^{L-1} y_{l}(n-i) \mathbf{h}[i]
$$

where $L$ is the length of the adaptive FIR filter $\mathbf{h}$.

The objective function is to minimize the energy of the output signal, that is the unconstrained optimization given by

$$
\underset{\mathbf{h}}{\operatorname{minimize}} y(n)^{2}=\left(y_{u}(n)-\sum_{i=0}^{L-1} y_{l}(n-i) \mathbf{h}[i]\right)^{2} .
$$


That is a quadratic function, the gradient is given explicitly by

$$
\mathbf{g}=-2 y(n) \mathbf{b}
$$

where $\mathbf{b}=\left[y_{l}(n), \ldots, y_{l}(n-L+1)\right]^{T}$. The gradient descent method can be applied to update $\mathbf{h}$ iteratively,

$$
\{\mathbf{h}\}_{n+1}=\{\mathbf{h}\}_{n}-\alpha_{n}\{\mathbf{g}\}_{n}
$$

where $\alpha_{n}$ is the step size and $\{.\}_{n}$ is the vector/matrix at $n$th iteration. For very small values of $\alpha_{n}$, the correction of $\{\mathbf{h}\}_{n}$ is small and the movement down the quadratic surface is slow, and as $\alpha_{n}$ is increased, the rate of descent increases. However, there is an upper limit on how large the step size may be. For values of $\alpha_{n}$ that exceed this limit, the trajectory of $\{\mathbf{h}\}_{n}$ becomes unstable and unbounded [32].

Taking the expected value of equation (17), we have

$$
E\left[\{\mathbf{h}\}_{n+1}\right]=E\left[\{\mathbf{h}\}_{n}\right]+2 \alpha_{n} E\left[\left(y_{u}(n)-\mathbf{b}^{T}\{\mathbf{h}\}_{n}\right) \mathbf{b}\right]
$$

or

$$
E\left[\{\mathbf{h}\}_{n+1}\right]=\left(\mathbf{I}-2 \alpha_{n} \mathbf{R}_{x}\right) E\left[\{\mathbf{h}\}_{n}\right]+2 \alpha_{n} \mathbf{r}_{u}
$$

where $\mathbf{R}_{x}=E\left[\mathbf{b b}^{T}\right]$ and $\mathbf{r}_{u}=E\left[y_{u}(n) \mathbf{b}\right]$.

Subtracting $\mathbf{h}$ from both sides of this equation, we have

$$
E\left[\{\mathbf{h}\}_{n+1}\right]-\mathbf{h}=\left(\mathbf{I}-2 \alpha_{n} \mathbf{R}_{x}\right) E\left[\{\mathbf{h}\}_{n}\right]+2 \alpha_{n} \mathbf{r}_{u}-\mathbf{h}
$$

Note that $\mathbf{h}$ is the solution of Wiener-Hopf equation, or $\mathbf{r}_{u}=$ $\mathbf{R}_{x} \mathbf{h}$, then

$$
\mathbf{c}_{n+1}=\left(\mathbf{I}-2 \alpha_{n} \mathbf{R}_{x}\right) \mathbf{c}_{n}
$$

where $\mathbf{c}_{n}=E\left[\{\mathbf{h}\}_{n}\right]-\mathbf{h}$ is the weight error vector at step $n$. It is clearly seen that if we select $\alpha_{n}$ to satisfy the maximum eigenvalue of matrix $\left(\mathbf{I}-2 \alpha_{n} \mathbf{R}_{x}\right)$ be less than 1, then $\{\mathbf{h}\}_{n+1}$ converges to $\mathbf{h}$.

In Fig. 5, we adopt the normalized least-mean-square (LMS) algorithm for the adaptive FIR filter, that is

$$
\{\mathbf{h}\}_{n+1}=\{\mathbf{h}\}_{n}+\frac{\beta}{\mathbf{b}^{T} \mathbf{b}} y(n) \mathbf{b},
$$

where $\beta$ is the normalized step size with $0<\beta<1$. With the normalization of the step size by $\mathbf{b}^{T} \mathbf{b}$, that avoids the gradient noise amplification when $\mathbf{b}$ is large [32].

The major problem of this structure is the 'signal leak through' [33] which may result in desired signal distortion. The 'signal leak through' occurs when the lower path does not complete suppress the desired signal and this can be problematic for broadband beamforming where it is difficult to ensure perfect signal cancellation across the frequency of interest [34]. In addition, the interference is partially suppressed in the final output, that happens when the lower path beampattern is not similar to the upper path beampattern's sidelobes, which leads to the interference in the upper path leaks to the final output.

To avoid misadjustment, filter coefficients should be updated only when the SIR is low [29] but estimating SIR is somewhat computationally expensive, e.g. using voice activity detection. In fact, our unconstrained problem has a special structure that can be utilized to estimate the SIR, that is, a ratio of energy between the lower path and upper path signals. Suppose that when the SOI is active or the SIR is high, the energy of the upper path signal dominates the energy of the lower path signal and vice versa (an example of this ratio is illustrated in Fig. 23). Then, we propose a rule to change the normalized step size: When the energy ratio is high, $\beta$ is increased and vice versa. Thus, the adaptive normalized step size $\beta_{a}$ is defined as

$$
\beta_{a}=\frac{\mathbf{b}^{T} \mathbf{b}}{\mathbf{b}_{u}^{T} \mathbf{b}_{u}} \beta,
$$

where $\mathbf{b}_{u}=\left[y_{u}(n), \ldots, y_{u}(n-L+1)\right]^{T}$ is a vector containing signals in the upper path. Then, we replace (18) by

$$
\{\mathbf{h}\}_{n+1}=\{\mathbf{h}\}_{n}+\frac{\beta}{\mathbf{b}_{\mathbf{u}}{ }^{T} \mathbf{b}_{\mathbf{u}}} y(n) \mathbf{b} .
$$

In case the energy of interference dominates the energy of $\mathrm{SOI}$, the formula (20) is better than the formula (18) since $\frac{\mathbf{b}^{T} \mathbf{b}}{\mathbf{b}_{u}^{T} \mathbf{b}_{u}}>1$ and it increases the convergence speed. So, we can use (20) to replace (18) to further improve the convergence rate of the filter coefficients, while still assuring the trajectory of $\{\mathbf{h}\}_{n}$ is stable.

The computation in (20) has the complexity of $\mathcal{O}(2 L)$, then total computational complexity of the proposed method is $\mathcal{O}\left(M L_{1}+M L_{2}+3 L\right)$ which is less than that of the GSC, $\mathcal{O}\left(M L_{1}+M L_{2}+3 M L\right)$.

From the beampattern subtracting pointview, for the instantaneous interferences, the gain and phase in the lower path beampattern are updated accordingly to synchronize to the gain and phase of critical sidelobes of the upper path beampattern. Updating is performed via the sense of minimizing the output signal's energy. Analogous to the GSC, this unconstrained LMS algorithm is the simplest form compared to other adaptive beamformings, e.g. Frost beamforming [21], LCMV or minimum variance distortionless response. Compared to the GSC, the computational complexity of this method is reduced, since only one adaptive FIR filter is used instead of $M-1$ adaptive FIR filters. Moreover, the proposed method aims to avoid the drawbacks of the GSC, then its performance is superior to the GSC that will be illustrated in the simulation section.

\section{Numerical Simulation}

\section{A. Fixed beamforming with suppressed sidelobes}

The white noise gain (WNG) shows the ability of the array to suppress the incoherence noise, such as self-noise, array imperfection, etc. That is given by [14],

$$
\mathcal{W}(\mathbf{w}(\omega))=\frac{\left|\mathbf{w}^{H}(\omega) \mathbf{d}\left(\omega, \theta_{0}\right)\right|^{2}}{\mathbf{w}^{H}(\omega) \mathbf{w}(\omega)}
$$

where $\mathbf{w}(\omega)$ is the weight vector of beamforming, $\mathbf{d}\left(\omega, \theta_{0}\right)$ is the steering vector of the look direction.

Another index used to evaluate the beampattern is the directivity factor (DF), it measures the ability to preserve the source of interest while suppressing the signal coming from other directions [14],

$$
\mathcal{D}(\mathbf{w}(\omega))=\frac{\left|\mathbf{w}(\omega)^{H} \mathbf{d}\left(\omega, \theta_{0}\right)\right|^{2}}{\mathbf{w}(\omega)^{H} \boldsymbol{\Gamma}(\omega) \mathbf{w}(\omega)^{H}}
$$


where $\Gamma(\omega)$ is the pseudo-coherence matrix of the diffuse noise field,

$$
\boldsymbol{\Gamma}(\omega)[i, j]=\operatorname{sinc}\left(\frac{\omega d_{i j}}{c}\right)
$$

where $d_{i j}$ is the distance between sensor $i$ and sensor $j, c$ is the wave speed.
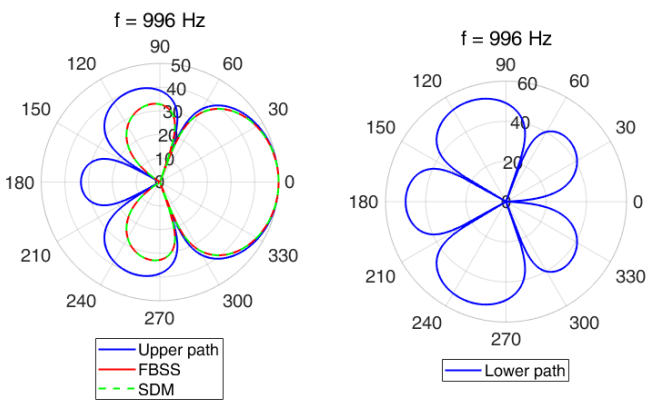

(a) $1 \mathrm{kHz}$.
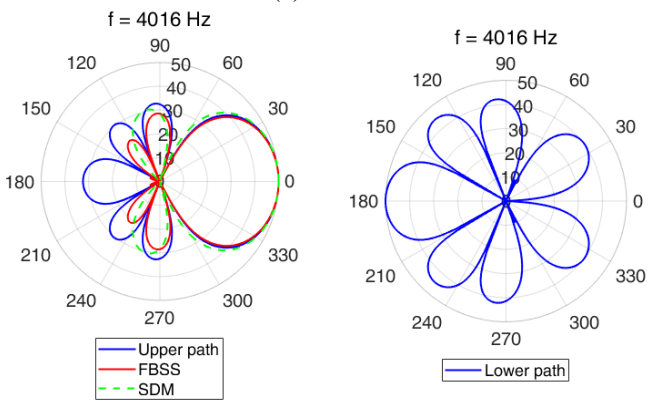

(b) $4 \mathrm{kHz}$.
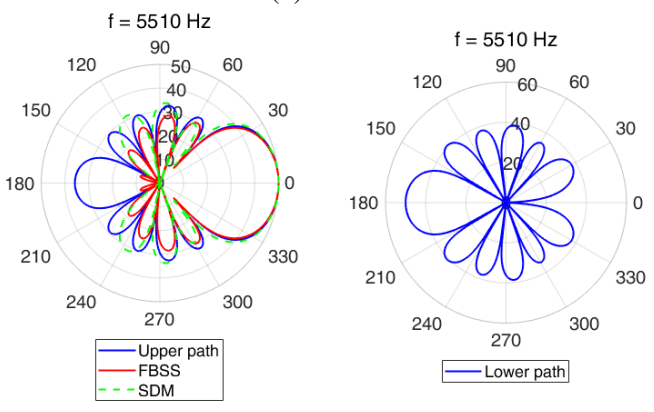

(c) $5.5 \mathrm{kHz}$

Fig. 6: Beampatterns at some frequencies: (left) upper path beampattern, FBSS and SDM, (right) lower path beampattern.

We simulated broadband beamforming for a uniform linear array of $M=7$ sensors with the inter-distance $d_{H}=0.02 \mathrm{~m}$, the reference sensor is at the middle of the array. The upper path beampattern has a look direction at $0^{\circ}$ and fixed nulls at $70^{\circ}, 150^{\circ}$. Then, the lower path beampattern has fixed nulls at $0^{\circ}, 70^{\circ}$ and $150^{\circ}$, and we set $\theta_{m}=180^{\circ}$. Due to spatial aliasing at high frequencies, there are some extra nulls at the upper path beampattern, we need to insert these nulls to the lower path beampattern as well. The noise model parameter $\mu$ in (7) is set 0.1 for all simulations.

In comparison, we also design superdirective beamforming with multiple constraints (SDM) with look direction at $0^{\circ}$ and fixed nulls at $70^{\circ}, 150^{\circ}$ and $180^{\circ}$. In Fig. 6, we plotted the beampatterns at $1 \mathrm{kHz}, 4 \mathrm{kHz}$ and $5.5 \mathrm{kHz}$, it is clear that sidelobes of the upper path beampattern are suppressed for all frequencies, especially the sidelobe contains $\theta_{m}=180^{\circ}$ is almost completely suppressed. In comparison with the SDM, both always assure the nulls at $70^{\circ}, 150^{\circ}$ and $180^{\circ}$, but the FBSS has smaller sidelobe regions at high frequencies, e.g., $4 \mathrm{kHz}$ and $5.5 \mathrm{kHz}$ in Figs $6 \mathrm{~b}$ and $6 \mathrm{c}$, respectively. Note that the number of nulls at Figs. $6 a, 6 b$ and $6 c$ are different, that causes the spatial aliasing effect.

Fig. 7, Fig. 8 and Fig. 9 plot the broad beampattern from $500 \mathrm{~Hz}$ to $6 \mathrm{kHz}$ for the upper path beampattern, lower path beampattern and FBSS's beampattern, respectively.

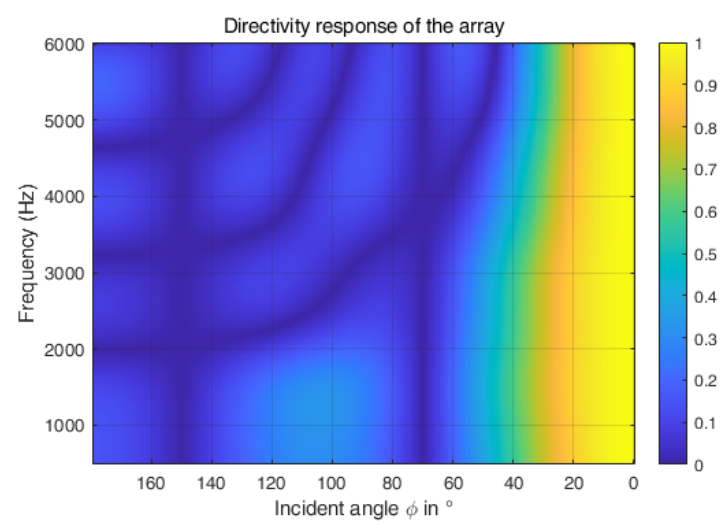

Fig. 7: The broadband beampattern of the upper path.

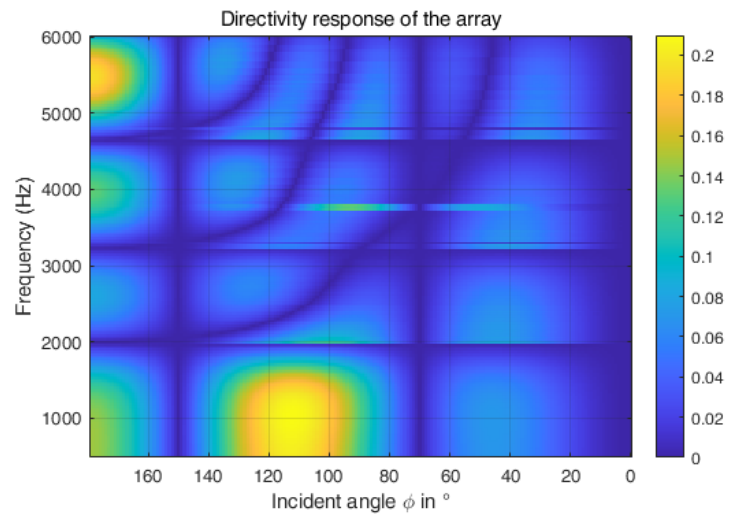

Fig. 8: The broadband beampattern of the lower path.

The WNG and DF of upper path beamforming, FBSS and SDM are plotted in Fig. 10. It is clearly seen that, the FBSS improves the DF compared to others, while the WNG is still in the acceptable range. Although the results of this simulation do not surprise us, i.e. a design with the regularization of the WNG and DF, the target of FBSS is frequency-invariant mainlobe and minimizing the sidelobes' region. This objective function is different with other approaches in the literature. Moreover, the proposed method enables to use multiple sensor arrays to form a single desired beam's shape, e.g. the upper path is an endfire array aims to build the mainlobe and the lower path is another linear array aims to reproduce the 


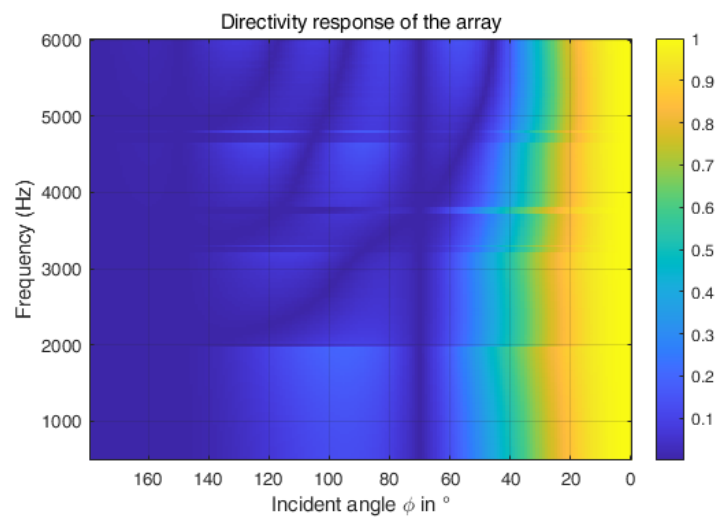

Fig. 9: The broadband beampattern of the FBSS.

sidelobes, in such a case, combining the beamforming of two arrays can be performed via FSBB.

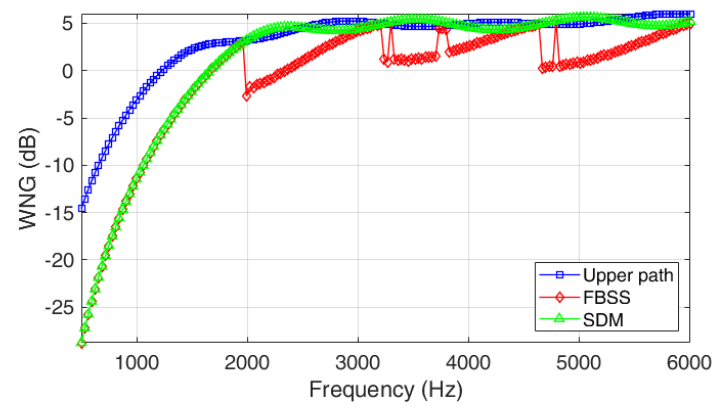

(a) WNG.

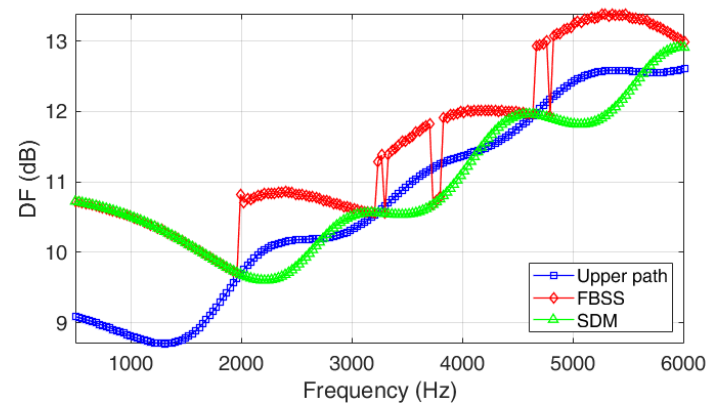

(b) DF.

Fig. 10: WNGs and DFs over frequency.

\section{B. Adaptive beamforming with suppressed sidelobes}

The signal-to-noise ratio (SNR) is defined as

$$
\mathrm{SNR}=10 \log _{10} \frac{\sigma_{s}^{2}}{\sigma_{n}^{2}}
$$

where $\sigma_{s}^{2}$ and $\sigma_{n}^{2}$ are the variances of SOI and additive noise, respectively.

There are two interferences impinging on the array that have variances $\sigma_{1}^{2}, \sigma_{2}^{2}$, then the SIR is defined as

$$
\mathrm{SIR}=10 \log _{10} \frac{\sigma_{s}^{2}}{\sigma_{1}^{2}+\sigma_{2}^{2}}
$$

To evaluate the performance of adaptive beamforming, we measure the signal's error (SE) between the SOI and beamforming output, that is

$$
\mathrm{SE}=E\left[(y(n)-s(n))^{2}\right]
$$

where $E[$.$] is the expectation value, y(n)$ is the beamforming signal and $s(n)$ is the SOI.

In the first simulation, we set $\mathrm{SNR}=10 \mathrm{~dB}$ and $\sigma_{1}^{2}=\sigma_{2}^{2}=$ $\sigma_{s}^{2}$, that is $\mathrm{SIR}=-3 \mathrm{~dB}$. The $\mathrm{SOI}$ at $0^{\circ}$ contains two impulse response signals, as shown in Fig. 12. Two interferences at $70^{\circ}$ and $150^{\circ}$ contain multi-tone signal and chirp signal, respectively.

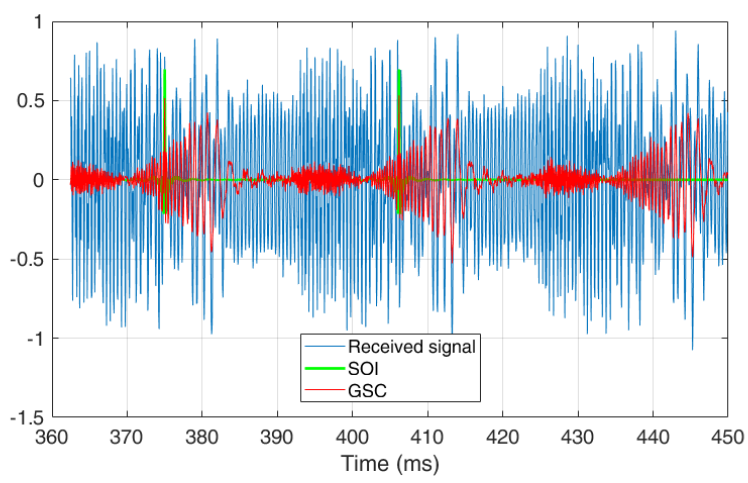

(a) GSC.

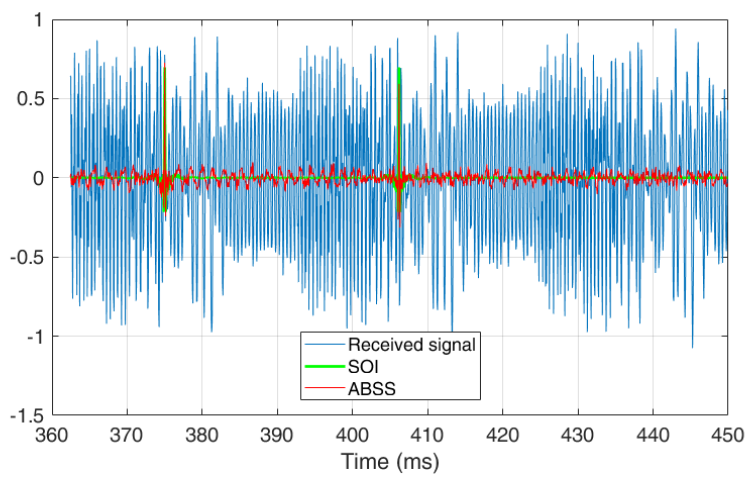

(b) ABSS.

Fig. 11: Free-field environment: Comparison between the GSC and ABSS.

1) Free-field environment: We set $L=511$ for both ABSS and GSC. For the beamformings inside ABSS, we use similar beamformings designed in Section IV-A for frequency range from $500 \mathrm{~Hz}$ to $6 \mathrm{kHz}$ and applying inverse discrete Fourier transfrom to obtain $\mathbf{W}_{l}, \mathbf{W}_{u}$ with $L_{1}=L_{2}=512, \beta$ is set 0.01 . The waveform of beamformings, measurement signal and SOI are plotted in Fig. 11. Both methods are able to suppress the noise, while preserving the SOI in the output beamformings.

More specifically, the comparisons of the ABSS and GSC from $312.5 \mathrm{~ms}$ to $450 \mathrm{~ms}$ are plotted in Fig. 11 and 12 where the SEs of GSC and ABSS are 0.0151 and 0.0011 , respectively.

Next, the SOI is simulated by summing a sinuous signal and two impulse response and the SIR is changed from $-30 \mathrm{~dB}$ to 


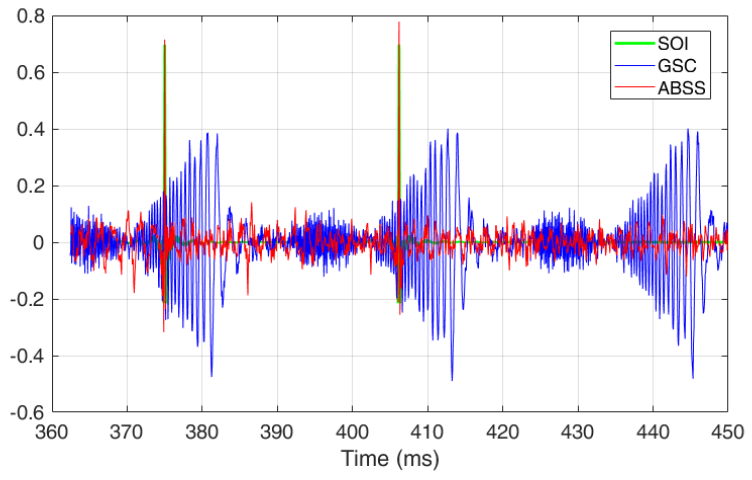

Fig. 12: Free-field environment: Comparison between the GSC and ABSS from $312.5 \mathrm{~ms}$ to $450 \mathrm{~ms}$.

$10 \mathrm{~dB}$, the SNR is set to $10 \mathrm{~dB}$ or $20 \mathrm{~dB}$. We measure the SEs over SIR for the GSC, FBSS and ABSS with 25 Monte Carlo trials. Fig. 13 shows that the ABSS outperforms the GSC in terms of preserving the SOI and suppressing interferences. It is worth noting that the computational complexity of ABSS is less than that of GSC.

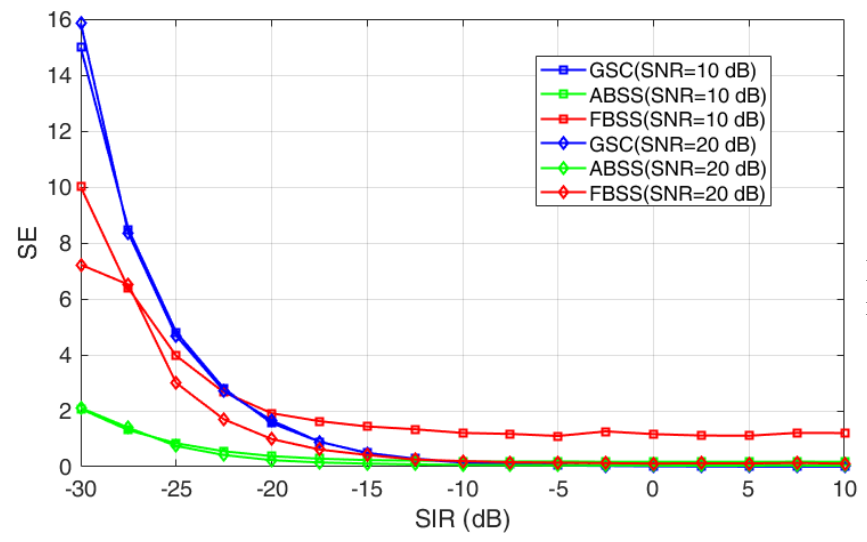

Fig. 13: Free-field environment: SEs over SIR for the ABSS, FBSS and GSC, two interferences at $90^{\circ}$ and $150^{\circ}$ (a null position).

Another example, the interferences come from $90^{\circ}$ and $180^{\circ}$ (the directions are different with nulls' direction of the designed beampatterns at $70^{\circ}$ and $150^{\circ}$ ), SEs over SIR are plotted in Fig. 14.

2) Reverberant environment: We simulate a room with dimensions of $3.5 \times 6 \times 3 \mathrm{~m}^{3}$ in which a uniform linear array is located $(x, y, z)=(1.5,2,1)$. The uniform linear array contains $M=7$ microphones with the inter-distance $d_{H}=2$ $\mathrm{cm}$ located along the X-axis. The SOI located at $(x, y, z)=$ $\left(1.5+0.5 \cos 0^{\circ}, 2+0.5 \sin 0^{\circ}, 1\right)$ contains pulse signal. Two interferences at $(x, y, z)=\left(1.5+0.5 \cos 70^{\circ}, 2+0.5 \sin 70^{\circ}, 1\right)$ and $(x, y, z)=\left(1.5+0.5 \cos 150^{\circ}, 2+0.5 \sin 150^{\circ}, 1\right)$ contain multi-tone signal (from $500 \mathrm{~Hz}$ to $6 \mathrm{kHz}$ ) and single-frequency signal $(2 \mathrm{kHz})$, respectively. We use a room impulse response (RIR) generator in [35] with reverberation time $T_{60}=300$ ms. In addition, the Gaussian noises with SNR $=10 \mathrm{~dB}$ are added to received signals.

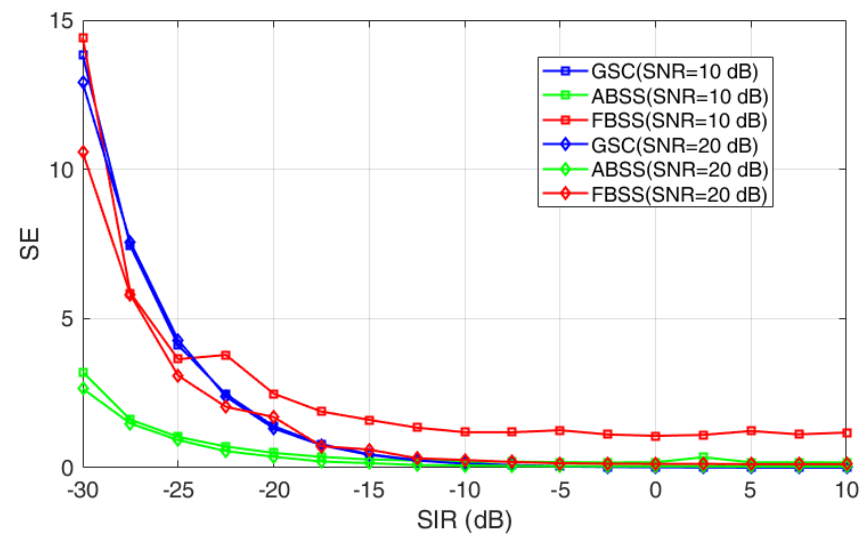

Fig. 14: Free-field environment: SEs over SIR for the ABSS, FBSS and GSC, two interferences at $90^{\circ}$ and $180^{\circ}$.

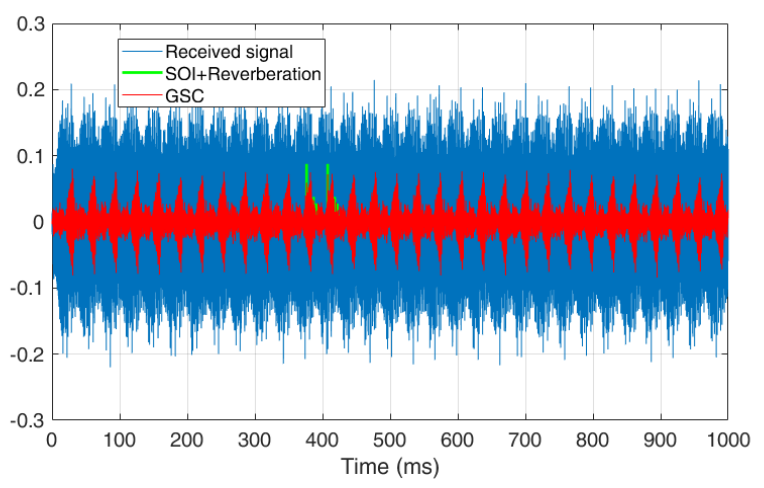

Fig. 15: Reverberant environment: The simulation for the GSC in a reverberant room and white noise $(\mathrm{SNR}=10 \mathrm{~dB})$.

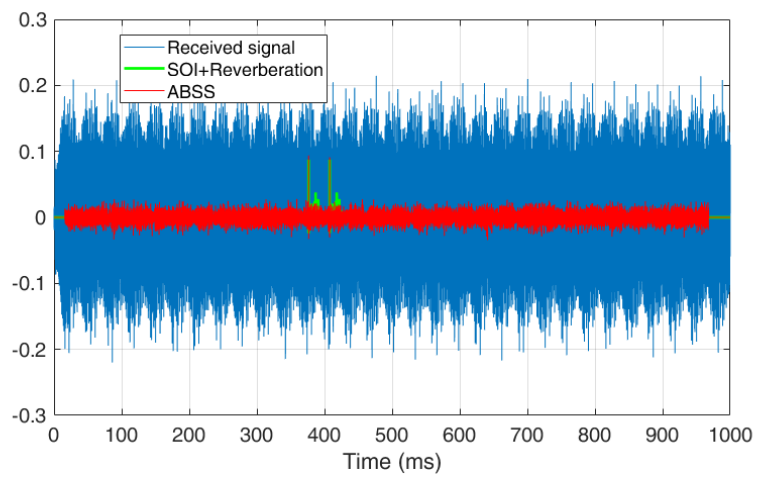

Fig. 16: Reverberant environment: The simulation for the ABSS in a reverberant room and white noise $(\mathrm{SNR}=10 \mathrm{~dB})$.

We set $L=511$ and $L_{1}=L_{2}=512$, Figs. 15 and 16 plot the output signals of GSC and ABSS, respectively. Comparing two results, it is clearly seen that the ABSS outperforms the GSC in terms of suppressing interferences.

In Fig. 17, the output of ABSS (adaptive beamforming) and FBSS (fixed beamforming) are compared and we also observe the out-performance of the ABSS, especially at the low frequencies where the WNG of FBSS is low, as shown in 
Fig. 10 .

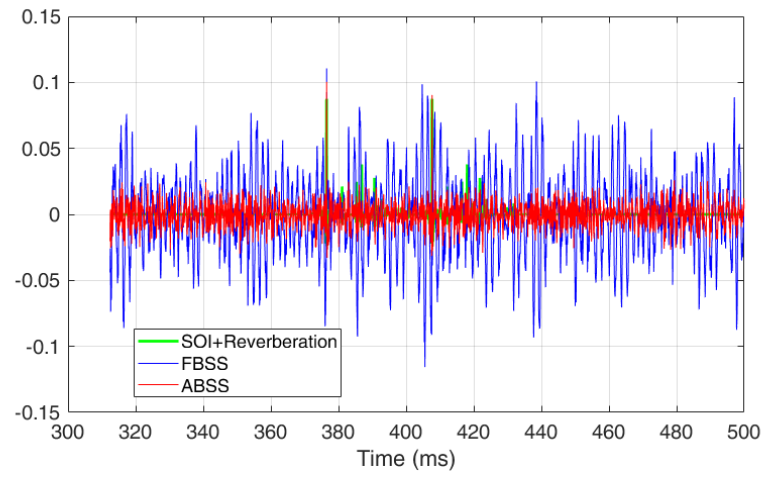

Fig. 17: Reverberant environment: Comparing the ABSS (adaptive beamforming) with the FBSS (fixed beamforming) in a reverberant room and white noise $(\mathrm{SNR}=10 \mathrm{~dB})$.

3) Diffuse environment: Similarly to the simulation in Section IV-B2, instead of white noise, we consider for the diffuse noise with $\mathrm{SNR}=10 \mathrm{~dB}$ in this simulation.

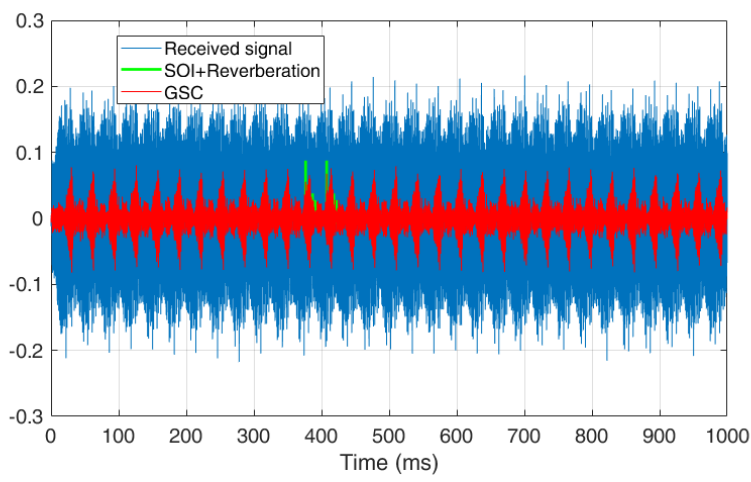

Fig. 18: The simulation for the GSC in a reverberant room and diffuse noise $(\mathrm{SNR}=10 \mathrm{~dB})$.

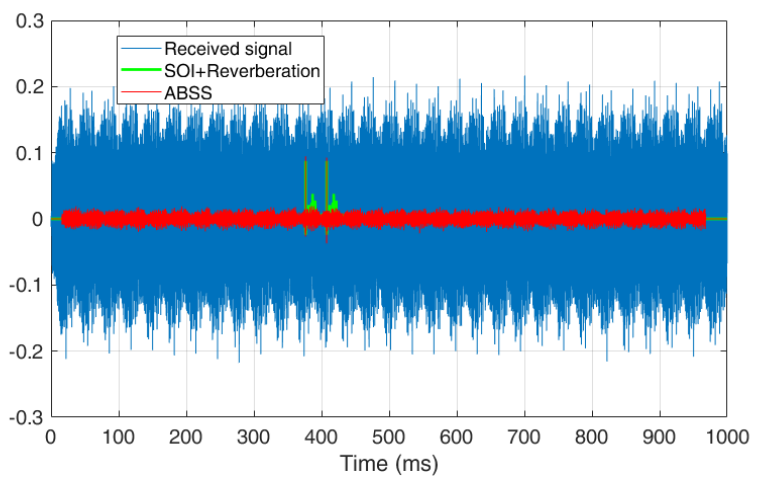

Fig. 19: The simulation for the ABSS in a reverberant room and diffuse noise $(\mathrm{SNR}=10 \mathrm{~dB})$.

In Figs. 18 and 19, we plot the results of GSC and ABSS respectively. We can observe that the ABSS is also tolerent to the diffuse noise. Similarly, in Fig. 20, the ABSS is superior to the FBSS.

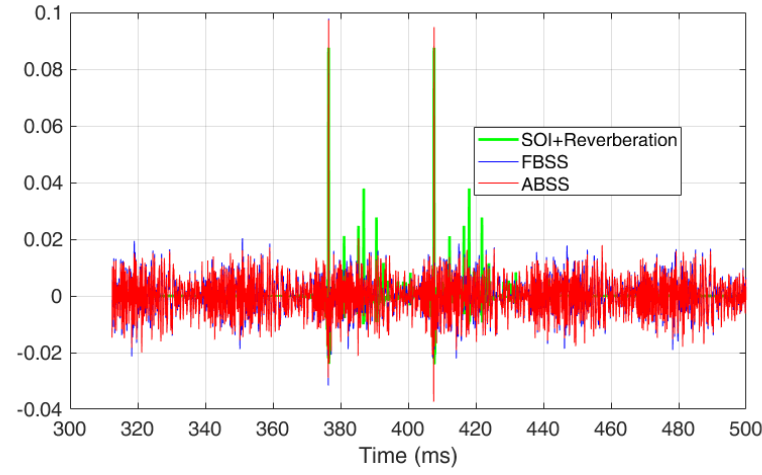

Fig. 20: Comparing the ABSS (adaptive beamforming) with the FBSS (fixed beamforming) in a reverberant room and diffuse noise (SNR $=10 \mathrm{~dB})$.

\section{EXPERIMENT}

We conduct an experiment which is similar to the simulation in Section IV-B2. An uniform linear array $(M=7$ sensors with the inter-distance $d_{H}=0.02 \mathrm{~m}$ ) was placed at the center of a reverberant room with dimension of $3.5 \times 6 \times 3 \mathrm{~m}^{3}$.

A person at the endfire direction of the array spoke into the microphone array for less than two seconds (within 4th and 5.5 th second), while a speaker at the broadside direction of the array played a music sound, referred to as interference.

With the setting of $L=511$ and $\beta=0.01$, the beamforming signal of the upper path is plotted in the top of Fig. 21, next is the beamforming signal of the lower path. For comparison, the ABSS's signal and upper path's signal are plotted in the bottom of Fig. 21. Also, their spectra are plotted in Fig. 22. The SIR of ABSS's signal is increased compared to the beamforming of the upper path.
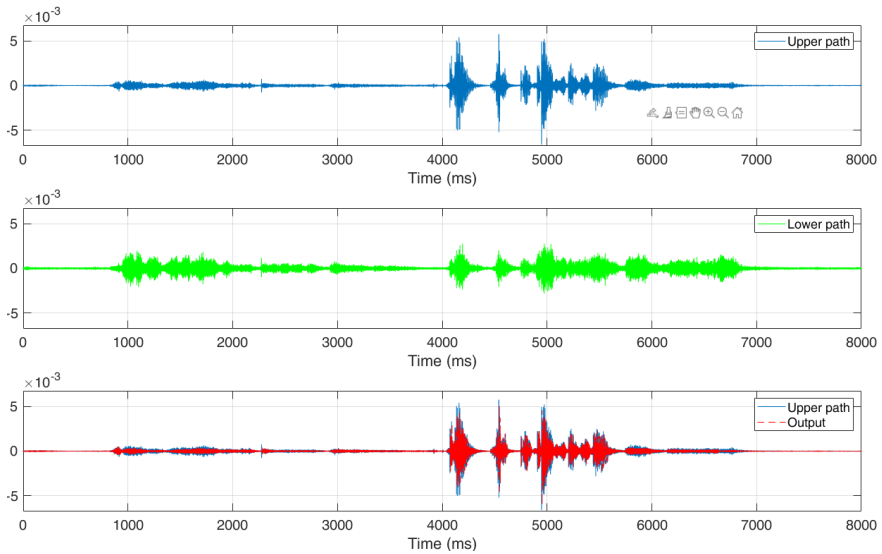

Fig. 21: Wave signals: (top) upper path's signal, (middle) lower path's signal and (bottom) ABSS's signal and upper path's signal. Within 4 th and 5.5th second, the source of interest is present and overlap with the interference.

In Fig, 23, the energy ratio between the lower path and upper path signals is plotted. As mentioned in Section III-B, this ratio is proportional to the step size of the adaptive filter: It is bigger when the energy of interference dominates that of SOI and vice versa. 


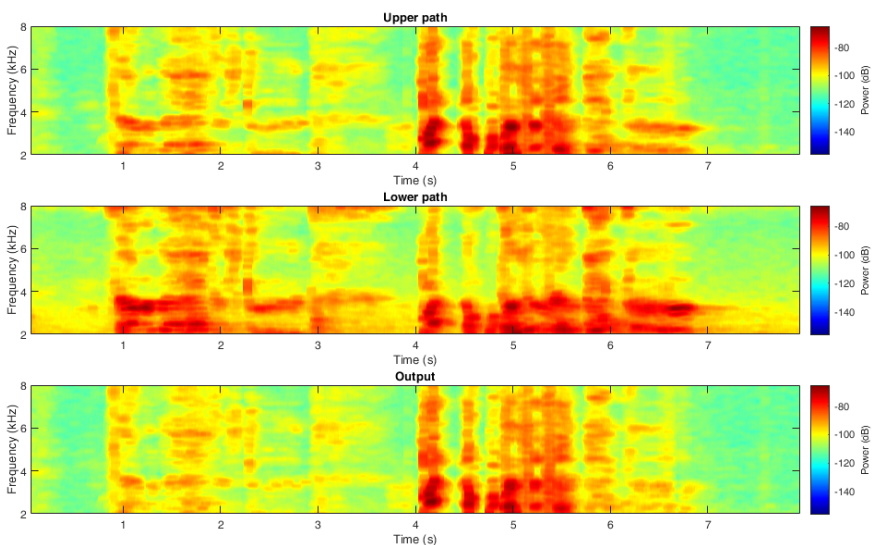

Fig. 22: Spectra:(top) upper path's signal, (middle) lower path's signal and (bottom) ABSS's signal. Within 4th and 5.5th second, the source of interest is present and overlap with the interference.

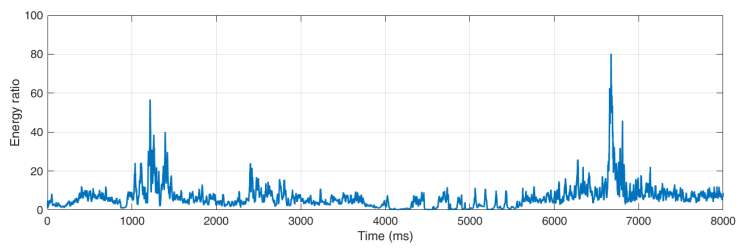

Fig. 23: The energy ratio between the lower path and upper path signals. Within 4th and 5.5th second, the source of interest is present and overlap with the interference

Besides, we performed informal subjective listening evaluations to compare the ABSS's signal and the upper path's signal. Our listeners noticed interference reductions in ABSS's signal and the distortion of the SOI was unnoticeable.

\section{CONCLUSION}

In this paper, we have proposed two alternative approaches to the generalized sidelobe canceler beamformer: One is for fixed beamforming, another is for adaptive beamforming. The proposed fixed beamforming applies for a symmetric array layout and the reference point must be at the center of the array. With that constraint, the difference of phase response between the lower path beamforming and the upper path beamforming is almost constant over the direction, so we can use a fixed scale number to compensate for the difference before subtracting. For the proposed adaptive beamforming, it can apply for an arbitrary array layout. As presented in the simulation section, this proposed method outperforms the generalized sidelobe canceler in terms of interference suppression and preserving the signal of interest. Moreover, the computational complexity of this method is less than that of the generalized sidelobe canceler because the proposed method uses one adaptive FIR filter, while the GSC uses $M-1$ adaptive FIR filters. In comparison to other adaptive designs, the problem of the proposed method is the unconstrained optimization and the beamformer coefficients are updated via the normalized least-mean square algorithm, that is much simpler than the constrained optimization-based methods, such as Frost beamforming, MVDR, LCMV, etc. For example, our real-time implementation for seven-microphone case only requires one processor, i.e., DSP56725 chip by NXP. Besides, the proposed method could extend the lower path to multiple lower paths where each path has a different beampattern and it may belong to different sensor arrays. That enables the ability to combine multiple arrays where one aims to build the mainlobe, others aim to suppress the sidelobes.

\section{APPENDIX A}

\section{PHASE RESPONSE OF BEAMFORMING}

Consider a complex number is defined by

$$
b_{m}=\left[\mathbf{a}_{m}^{H}, 1, \mathbf{a}_{m}^{T}\right] \mathbf{R}\left[\mathbf{a}^{T}, 1, \mathbf{a}^{H}\right]^{T}
$$

where $\left[\mathbf{a}_{m}^{H}, 1, \mathbf{a}_{m}^{T}\right]$ is the row $m$ of the matrix $\mathbf{D}_{u}^{T}$ and $\mathbf{R}=$ $(\boldsymbol{\Gamma}+\mu \mathbf{I})^{-1}$.

First, we prove for the case $\mu=0$, then $\mathbf{R}=\Gamma^{-1}$. Note that $\boldsymbol{\Gamma}(\omega)$ is the pseudo-coherence matrix of the diffuse noise field

$$
\boldsymbol{\Gamma}(\omega)[i, j]=\operatorname{sinc}\left(\frac{\omega d_{i j}}{c}\right),
$$

and the array is symmetric then $\boldsymbol{\Gamma}$ has a special structure, i.e,

$$
\boldsymbol{\Gamma}=\left[\begin{array}{ccc}
\boldsymbol{\Gamma}_{1} & \gamma & \boldsymbol{\Gamma}_{2} \\
\gamma & 1 & \gamma \\
\boldsymbol{\Gamma}_{2} & \gamma & \boldsymbol{\Gamma}_{1}
\end{array}\right]
$$

Moreover, $\boldsymbol{\Gamma}$ is a symmetric matrix, it can be decomposed as

$$
\Gamma=\mathbf{V D V}^{-1}
$$

where $\mathbf{V}$ is an unitary matrix $\left(\mathbf{V}^{-1}=\mathbf{V}^{T}\right)$ formed by eigenvectors of $\boldsymbol{\Gamma}$ and $\mathbf{D}$ is diagonal matrix of eigenvalues. So, we have

$$
\boldsymbol{\Gamma}^{-1}=\mathbf{V D}^{-1} \mathbf{V}^{-1}
$$

That implies $\boldsymbol{\Gamma}^{-1}$ has similar structure with $\boldsymbol{\Gamma}$, then $\mathbf{R}$ can be presented by

$$
\mathbf{R}=r\left[\begin{array}{ccc}
\mathbf{R}_{1} & \mathbf{r} & \mathbf{R}_{2} \\
\mathbf{r} & 1 & \mathbf{r} \\
\mathbf{R}_{2} & \mathbf{r} & \mathbf{R}_{1}
\end{array}\right]
$$

where $r$ is a real number used to scale the center's value of matrix to one, $\mathbf{r}$ is the real-valued vector and $\mathbf{R}_{1}, \mathbf{R}_{2}$ are the real-valued matrix. Thus,

$$
\begin{aligned}
b_{m} / r= & \mathbf{a}_{m}^{H} \mathbf{R}_{1} \mathbf{a}+\mathbf{a}_{m}^{H} \mathbf{r}+\mathbf{a}_{m}^{H} \mathbf{R}_{2} \mathbf{a}^{*} \\
& +\mathbf{a}^{T} \mathbf{r}+1+\mathbf{a}^{H} \mathbf{r} \\
& +\mathbf{a}_{m}^{T} \mathbf{R}_{2} \mathbf{a}+\mathbf{a}_{m}^{T} \mathbf{r}+\mathbf{a}_{m}^{T} \mathbf{R}_{1} \mathbf{a}^{*} \\
=1 & +2 \operatorname{Re}\left\{\mathbf{a}_{m}^{H} \mathbf{R}_{1} \mathbf{a}\right\}+2 \operatorname{Re}\left\{\mathbf{a}_{m}^{H} \mathbf{R}_{2} \mathbf{a}^{*}\right\} \\
& +2 \operatorname{Re}\left\{\mathbf{a}_{m}^{T} \mathbf{r}\right\}+2 \operatorname{Re}\left\{\mathbf{a}^{T} \mathbf{r}\right\}
\end{aligned}
$$

(where (.)* is the conjugate operator, $\operatorname{Re}\{$.$\} is the real$ part of a complex number) is a real number. It leads to $\left[\mathbf{A}_{u}^{H}, \mathbf{1}, \mathbf{A}_{u}^{T}\right](\boldsymbol{\Gamma}+\mu \mathbf{I})^{-1}\left[\mathbf{a}^{T}, 1, \mathbf{a}^{H}\right]^{T}$ is the real-valued vector and $\left[\mathbf{A}_{u}^{H}, \mathbf{1}, \mathbf{A}_{u}^{T}\right](\boldsymbol{\Gamma}+\mu \mathbf{I})^{-1}\left[\mathbf{A}_{u}^{T}, \mathbf{1}, \mathbf{A}_{u}^{H}\right]^{T}$ is the real-valued matrix. As a result, $\mathcal{B}_{u}(\theta)$ in (12) is a real number. 
For the case $\mu \neq 0$, applying Woodbury's formula [36], we have

$$
\begin{aligned}
\mathbf{R} & =\left(\mu \mathbf{I}+\mathbf{V} \mathbf{D} \mathbf{V}^{-1}\right)^{-1} \\
& =\frac{1}{\mu} \mathbf{I}-\frac{1}{\mu} \mathbf{V}\left(\mathbf{D}^{-1}+\frac{1}{\mu} \mathbf{V}^{-1} \mathbf{V}\right) \frac{1}{\mu} \mathbf{V}^{-1} \\
& =\frac{1}{\mu} \mathbf{I}-\frac{1}{\mu^{2}}\left(\mathbf{V D}^{-1} \mathbf{V}^{-1}+\frac{1}{\mu} \mathbf{I}\right) \\
& =\left(\frac{1}{\mu}-\frac{1}{\mu^{3}}\right) \mathbf{I}-\frac{1}{\mu^{2}} \boldsymbol{\Gamma}^{-1}
\end{aligned}
$$

has a similar structure with $\Gamma^{-1}$, then $\mathcal{B}_{u}(\theta)$ in (12) is also a real number.

\section{REFERENCES}

[1] H. Krim and M. Viberg, "Two decades of array signal processing research: the parametric approach," IEEE signal processing magazine, vol. 13, no. 4, pp. 67-94, 1996.

[2] R. J. Mailloux, Phased array antenna handbook. Artech house, 2017.

[3] M. Brandstein and D. Ward, Microphone arrays: signal processing techniques and applications. Springer Science \& Business Media, 2001.

[4] J. Benesty, J. Chen, and Y. Huang, Microphone array signal processing. Springer Science \& Business Media, 2008, vol. 1.

[5] A. Richard Thompson, J. M. Moran, and G. W. Swenson Jr, Interferometry and synthesis in radio astronomy. Springer Nature, 2017.

[6] M. Simeoni and J.-A. Martin, "Towards more accurate and efficient beamformed radio interferometry imaging," Tech. Rep., 2015.

[7] S. Haykin, "Array signal processing," Englewood Cliffs, 1985.

[8] L. C. Godara, "Application of antenna arrays to mobile communications. ii. beam-forming and direction-of-arrival considerations," Proceedings of the IEEE, vol. 85, no. 8, pp. 1195-1245, 1997.

[9] A. J. Paulraj and C. B. Papadias, "Space-time processing for wireless communications," IEEE signal processing magazine, vol. 14 , no. 6 , pp. 49-83, 1997.

[10] P. Hurley and M. Simeoni, "Flexibeam: analytic spatial filtering by beamforming," in 2016 IEEE International Conference on Acoustics, Speech and Signal Processing (ICASSP). Ieee, 2016, pp. 2877-2880.

[11] B. Rafaely, Fundamentals of spherical array processing. Springer, 2015, vol. 8.

[12] T. Van and H. L, Optimum array processing: Part IV of detection, estimation, and modulation theory. John Wiley \& Sons, 2004.

[13] G. W. Elko, "Superdirectional microphone arrays," in Acoustic signal processing for telecommunication. Springer, 2000, pp. 181-237.

[14] J. Bitzer and K. U. Simmer, "Superdirective microphone arrays," in Microphone arrays. Springer, 2001, pp. 19-38.

[15] G. W. Elko, "Differential microphone arrays," in Audio signal processing for next-generation multimedia communication systems. Springer, 2004, pp. 11-65.

[16] J. Benesty and C. Jingdong, Study and design of differential microphone arrays. Springer Science \& Business Media, 2012, vol. 6.

[17] H. Teutsch and G. W. Elko, "First- and second-order adaptive differential microphone arrays," The Journal of the Acoustical Society of America, vol. 115, no. 1, pp. 57-60, 2004.

[18] M. Buck, "Aspects of first-order differential microphone arrays in the presence of sensor imperfections," Eur. Trans. Telecommun., vol. 13, no. 2, pp. 115-122, March 2002

[19] L. Zhao, J. Benesty, and J. Chen, "Design of robust differential microphone arrays," IEEE/ACM Trans. Audio, Speech, Lang. Process, vol. 22, no. 10, pp. 1455-1464, October 2014.

[20] C. Pan, J. Benesty, and J. Chen, "Design of robust differential microphone arrays with orthogonal polynomials," The Journal of the Acoustical Society of America, vol. 138, no. 2, pp. 1079-1089, August 2015.

[21] O. Forst, "An algorithm for linearly constrained adaptive processing," Proc. IEEE, vol. 60, no. 8, pp. 926-935, 1972.

[22] G. Doblinger, "Optimization of wideband fixed beamformers with adaptive sensor calibration," in 2010 18th European Signal Processing Conference. IEEE, 2010, pp. 2062-2066.

[23] M. Souden, J. Benesty, and S. Affes, "A study of the 1cmv and mvdr noise reduction filters," IEEE Transactions on Signal Processing, vol. 58, no. 9, pp. 4925-4935, 2010.

[24] L. Griffiths and C. Jim, "An alternative approach to linearly constrained adaptive beamforming," IEEE Transactions on antennas and propagation, vol. 30, no. 1, pp. 27-34, 1982.
[25] S. E. Nordholm, H. H. Dam, C. C. Lai, and E. A. Lehmann, "Broadband beamforming and optimization," in Academic Press Library in Signal Processing. Elsevier, 2014, vol. 3, pp. 553-598.

[26] I. Claesson and S. Nordholm, "A spatial filtering approach to robust adaptive beaming," IEEE Transactions on Antennas and Propagation, vol. 40, no. 9, pp. 1093-1096, 1992.

[27] S. Nordholm, I. Claesson, and B. Bengtsson, "Adaptive array noise suppression of handsfree speaker input in cars," IEEE Transactions on Vehicular Technology, vol. 42, no. 4, pp. 514-518, 1993.

[28] S. Nordebo, I. Claesson, and S. Nordholm, "Adaptive beamforming: spatial filter designed blocking matrix," IEEE Journal of Oceanic Engineering, vol. 19, no. 4, pp. 583-590, 1994.

[29] O. Hoshuyama, A. Sugiyama, and A. Hirano, "A robust adaptive beamformer for microphone arrays with a blocking matrix using constrained adaptive filters," IEEE Transactions on signal processing, vol. 47, no. 10, pp. 2677-2684, 1999.

[30] S. Gannot, D. Burshtein, and E. Weinstein, "Signal enhancement using beamforming and nonstationarity with applications to speech," IEEE Transactions on Signal Processing, vol. 49, no. 8, pp. 1614-1626, 2001.

[31] S. L. Gay and J. Benesty, Acoustic signal processing for telecommunication. Springer Science \& Business Media, 2012, vol. 551.

[32] M. H. Hayes, Statistical digital signal processing and modeling. John Wiley \& Sons, 2009.

[33] B. Widrow, J. R. Glover, J. M. McCool, J. Kaunitz, C. S. Williams, R. H. Hearn, J. R. Zeidler, J. E. Dong, and R. C. Goodlin, "Adaptive noise cancelling: Principles and applications," Proceedings of the IEEE, vol. 63, no. 12, pp. 1692-1716, 1975.

[34] I. A. McCowan, "Robust speech recognition using microphone arrays," Ph.D. dissertation, Queensland University of Technology, 2001.

[35] E. A. Habets, "Room impulse response generator," Technische Universiteit Eindhoven, Tech. Rep, vol. 2, no. 2.4, p. 1, 2006.

[36] M. A. Woodbury, "Inverting modified matrices," Memorandum report, vol. 42 , no. 106 , p. $336,1950$. 